\section{Commentary: Can we pump our way out of heart failure with preserved ejection fraction? Not}

\section{so soon}

\author{
Nader Moazami, MD, and Deane Smith, MD
}

Heart failure with preserved ejection fraction (HFpEF) affects approximately one-half of patients with heart failure and contributes to the large burden of health care resources used. The significant rising trend in HFpEF is largely attributed to an increasing number of patients older than age 65 years with sedentary lifestyles, obesity, and hypertension in the community. ${ }^{1}$ Recent studies have suggested that $\mathrm{HFpEF}$ is characterized by abnormalities in both systolic and diastolic dysfunction, chronotropic incompetence, right ventricular dysfunction, impairments in endothelial function, and pulmonary vascular dysfunction. ${ }^{2}$ Many patients also have atrial fibrillation. Although diastolic dysfunction is a prominent feature in about two-thirds of patients, the pathophysiology is much more complex. Recent classifications suggest that known cardiomyopathies (eg, hypertrophic and infiltrative cardiomyopathies) are distinct entities, and although they share the common feature of diastolic dysfunction, they are not the same as HFpEF. ${ }^{3}$

Despite significant progress related to the treatment of heart failure with reduced ejection fraction, especially in the area of advances in mechanical circulatory support, there is presently no effective therapy for HFpEF. Fukamachi and colleagues ${ }^{4}$ designed a continuous flow pump for the mitral position-the left atrial assist device - and use this pump in an in vitro mock circulatory system. The experimental design is configured to mimic diastolic dysfunction with associated reduced left ventricle cavity size and volume. Overall, the best hemodynamic parameters are obtained

\footnotetext{
From the Department of Cardiothoracic Surgery, NYU Langone Health, New York, NY.

Disclosure: Authors have nothing to disclose with regard to commercial support.

Received for publication Feb 4, 2020; accepted for publication Feb 4, 2020; available ahead of print Feb 14, 2020.

Address for reprints: Nader Moazami, MD, Department of Cardiothoracic Surgery, NYU Langone Health, 530 First Ave, Suite 9V, New York, NY 10016 (E-mail: nader.moazami@nyulangone.org).

J Thorac Cardiovasc Surg 2021;162:129-30

$0022-5223 / \$ 36.00$

Copyright (c) 2020 by The American Association for Thoracic Surgery

https://doi.org/10.1016/j.jtcvs.2020.02.011
}

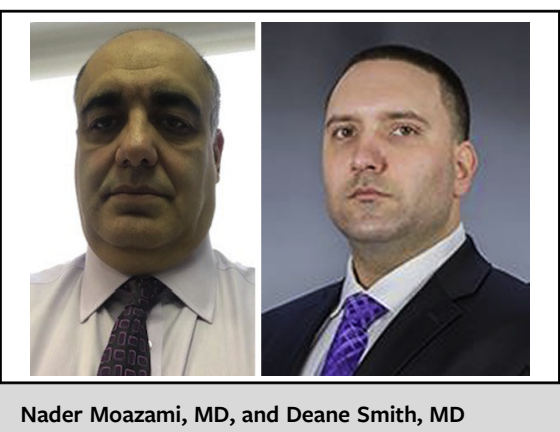

\section{CENTRAL MESSAGE \\ Significant gaps exists for the practical treatment of HFpEF with current pump technology.}

with the device in the mitral position and outflow to the left ventricle. The increasing degree of experimentally induced diastolic dysfunction (diastolic heart failure grade 1-3 with increasing severity) does not seem to have an influence on cardiac output, mean aortic pressure, or left atrial pressure, suggesting that the pump, in essence, is pushing blood through the left ventricle cavity and overcoming the limitations induced by diastolic dysfunction.

Although the experimental design is elegant as proof of concept, significant limitations exist in a mock circulatory loop that requires a pause in enthusiasm for the practicality of this approach being applied to actual patient care. First, the limitation of mechanical circulatory support as a standard left ventricular assist device configuration in the clinical setting in this patient population has taught us that pathophysiology is much more complicated. The primary limitation involves adequate output from the right side of the circulation, which may be impaired due to right ventricular systolic and diastolic dysfunction coupled with pulmonary vascular dysregulation. As long as we have a fixed right-sided output, the influence of any pump on the left side will be limited and the likelihood that cardiac output will increase with increasing pump speed is a fallacy. Most likely, a pump on the left side will reduce left atrial pressure and improve symptoms of dyspnea, but will have a fixed output that may or may not be sufficient for a surgical procedure of this magnitude. Second, to place a pump in the mitral position with a driveline somehow exiting the left atrium is asking for a multitude of complications, including thromboembolic events that are difficult to evaluate in a mock circulatory loop. Unfortunately, this elegant experimental design is far from the clinical application of pumping our way out of $\mathrm{HFpEF}$. 


\section{References}

1. Bhatia RS, Tu JV, Lee DS, Austin PC, Fang J, Haouzi A, et al. Outcome of heart failure with preserved ejection fraction in a population-based study. $N$ Engl J Med. 2006;355:260-9

2. Borlaug BA. Mechanisms of exercise intolerance in heart failure with preserved ejection fraction. Circ J. 2013;78:20-32.
3. Andersen MJ, Borlaug BA. Heart failure with preserved ejection fraction: current understandings and challenges. Curr Cardiol Rep. 2014;16:501.

4. Fukamachi K, Horvath DJ, Karimov JH, Kado Y, Miyamoto T, Kuban BD, et al. Left atrial assist device to treat patients with heart failure with preserved ejection fraction: initial in vitro study. J Thorac Cardiovasc Surg. 2021;162: $120-6$ 